\title{
Global and local disturbances in the magnetotail during reconnection
}

\author{
T. V. Laitinen ${ }^{1}$, R. Nakamura ${ }^{2}$, A. Runov ${ }^{2}$, H. Rème ${ }^{3}$, and E. A. Lucek \\ ${ }^{1}$ Finnish Meteorological Institute, Space Research, PL 503, 00101 Helsinki, Finland \\ ${ }^{2}$ Space Research Institute, Austrian Academy of Sciences, 8042 Graz, Austria \\ ${ }^{3}$ Centre d'Etude Spatiale des Rayonnements, Toulouse, France \\ ${ }^{4}$ The Blackett Laboratory, Imperial College, London, UK
}

Received: 11 December 2006 - Revised: 13 March 2007 - Accepted: 13 April 2007 - Published: 8 May 2007

\begin{abstract}
We examine Cluster observations of a reconnection event at $x_{\mathrm{GSM}}=-15.7 R_{E}$ in the magnetotail on $11 \mathrm{Oc}$ tober 2001, when Cluster recorded the current sheet for an extended period including the entire duration of the reconnection event. The onset of reconnection is associated with a sudden orientation change of the ambient magnetic field, which is also observed simultaneously by Goes- 8 at geostationary orbit. Current sheet oscillations are observed both before reconnection and during it. The speed of the flapping motions is found to increase when the current sheet undergoes the transition from quiet to active state, as suggested by an earlier statistical result and now confirmed within one single event. Within the diffusion region both the tailward and earthward parts of the quadrupolar magnetic Hall structure are recorded as an $\mathrm{x}$-line passes Cluster. We report the first observations of the Hall structure conforming to the kinks in the current sheet. This results in relatively strong fluctuations in $B_{z}$, which are shown to be the Hall signature tilted in the $y z$ plane with the current sheet.
\end{abstract}

Keywords. Magnetospheric physics (Magnetospheric configuration and dynamics; Magnetotail) - Space plasma physics (Magnetic reconnection)

\section{Introduction}

The magnetotail is a natural laboratory for magnetic reconnection. The antiparallel lobe fields set up the stage for the simplest possible reconnection geometry; however, the reconnection process need not choose the most simple form of a large-scale two-dimensional $\mathrm{x}$-line, and many observations indicate that it indeed does not. To name some examples, there are reports on off-centre and bifurcated current sheet profiles (Hoshino et al., 1996; Asano et al., 2003; Runov

Correspondence to: T. V. Laitinen

(tiera.laitinen@fmi.fi) et al., 2005; Thompson et al., 2006), current sheet flapping motion (Lui et al., 1978; Sergeev et al., 2003, 2004), multiple x-lines (Deng et al., 2004; Eastwood et al., 2005), and bursty bulk flows (Baumjohann et al., 1990) that can be interpreted as signs of localized reconnection events (Shay et al., 2003) or transient reconnection events (Sergeev et al., 1987).

From the global point of view reconnection is an important piece in the puzzle of magnetospheric dynamics. Tail reconnection is recognized as the main energy source of substorms (Baker et al., 1996): The substorm sequence commences with a growth phase, during which magnetic flux is added to the tail and the near-Earth current sheet grows thinner. At substorm onset a neutral line forms in the tail, most frequently at a radial distance of $20-30 R_{E}$ but sometimes also closer to the Earth (Nagai, 2006, and references therein). Global MHD simulations support the view that the reconnection site acts as a centre of Poynting flux focussing, where the incoming electromagnetic energy is partly converted to kinetic and thermal energy of the plasma and where some of it is diverted earthward (Laitinen et al., 2005). However, debate continues on whether tail reconnection should be regarded more as a "primus motor" or as a mediator and whether the energy it releases comes from the energy storage of the tail or as non-delayed Poynting flux from the magnetopause (Pulkkinen et al., 2006).

As reconnection proceeds, closed magnetic field lines in the plasma sheet are reconnected to form loops tailward of the $\mathrm{x}$-line. The loops form a plasmoid, which is released when reconnection reaches open field lines (Hones et al., 1984). Also multiple plasmoids have been observed (Belehaki et al., 1998). Earthward of the x-line the magnetic field geometry is observed to change towards a more dipolar form. A common ionospheric feature associated with near-Earth reconnection is the westward auroral electrojet. It is a consequence of the substorm current wedge of field-aligned currents, which is formed when some of the cross-tail current is diverted to the ionosphere.

Published by Copernicus GmbH on behalf of the European Geosciences Union. 

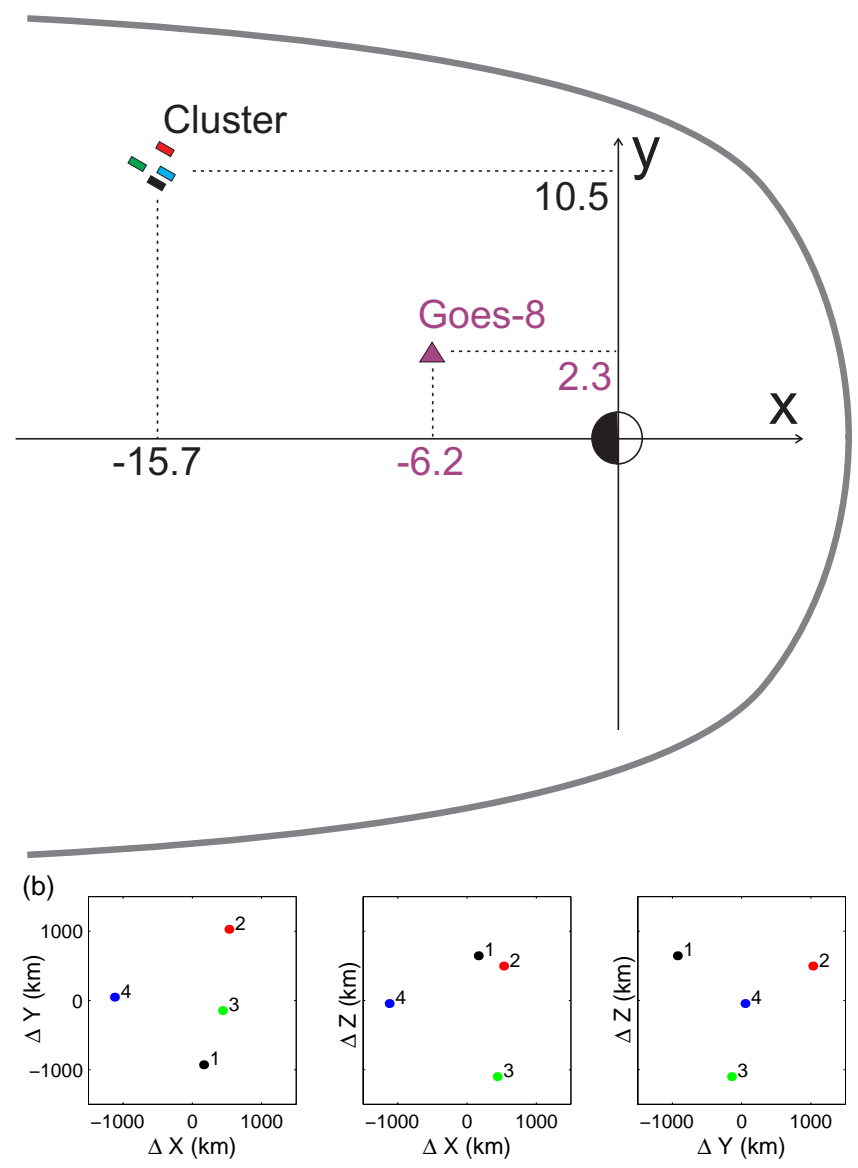

Fig. 1. (a) Cluster and Goes- 8 satellites in the magnetosphere at 03:30 UT on 11 October 2001. In GSM coordinates their locations were: Cluster at $[-15.7,10.5,2.5] R_{E}$ and Goes-8 at $[-6.2,2.3,-0.7] R_{E}$. The magnetopause drawn is a sketch only. Earth and the satellites are not to scale. (b) The relative locations of the four Cluster spacecraft. Cluster 1 is black, C2 red, C3 green and $\mathrm{C} 4$ blue. The figures are centered at the barycentre of the tetrahedron.

In this article we examine Cluster observations of an event which on the first glimpse appears as a classic example of a tailward-retreating x-line, but on closer examination shows several intriguing features: In Sect. 2 we introduce the event and show that a large-scale orientation change of the ambient magnetic field takes place when the reconnection starts. In the Discussion (Sect. 5) we suggest that this may reflect a global change in the magnetotail geometry. In Sect. 3 we find an $\mathrm{x}$-line from the tailward edge of the diffusion region; some possible explanations to this asymmetry are also given in the Discussion. The event also includes strong flapping motion of the current sheet and rapid fluctuations in the $y$ and z-components of the magnetic field. Cluster was fortunate enough to record the current sheet for a long period including the whole duration of the reconnection event, which allows us to compare the flapping motions at the same place during quiet and active times. This is described in Sect. 4; in addition, we show that the Hall fields conform to the kinks in the current sheet, which explains at least some of the largest reversals in $B_{z}$ within the diffusion region.

\section{Large-scale features}

\subsection{An overview of the event}

We examine the interval 03:00-04:00 UT on 11 October 2001, when Cluster observed a flow reversal and several current sheet crossings in the duskside flank of the magnetosphere. Several other ground-based and geostationary instruments recorded signatures of moderate activity. We will show that the reconnection onset occurred approximately at 03:25 UT; at that time the Cluster tetrahedron was located at $[x, y, z]=[-15.7,10.5,2.5] R_{E}$ in GSM coordinates. The positions of the spacecraft are shown in Fig. 1. Data from the Cluster magnetic field instrument FGM (Balogh et al., 2001) and ion spectrometer CIS (Rème et al., 2001) are used in this study.

Panels (a-d) in Fig. 2 show the solar wind conditions during the event period and during the preceding hour. The data are from the ACE spacecraft at the L1 libration point, but they have been time-shifted by $65 \mathrm{~min}$. The plots thus show the time evolution of the parameters at Earth assuming that the solar wind propagated unchanged from L1 at a speed of $380 \mathrm{~km} / \mathrm{s}$. This approximation is reasonable because the solar wind was quite steady during the period (panels $\mathrm{b}$ and $\mathrm{c}$ ). The moderate velocity and density combine to a relatively small dynamic pressure of about $1 \mathrm{nPa}$ (panel d). The magnetic field (panel a) had an almost constant magnitude of $5 \mathrm{nT}$ during the entire period and was directed duskward most of the time. Its z-component fluctuated around zero. Therefore the reconnection event was not preceded by enhanced energy loading into the magnetosphere. This is confirmed by the ground magnetometer (panel e) and geostationary energetic electron (f) data, which do not show any sign of a substorm growth phase preceding the reconnection onset time.

Figure 3 shows the magnetic field and proton velocity components measured by Cluster 4 from 03:00 to 04:00 UT. An eye-catching signature of reconnection are the two fast plasma flows, first tailward at 03:25-03:31 and then earthward at 03:36-03:43. Proton distribution functions (not shown) confirm that during the periods of fastest flow (03:28-03:30 and 03:39-03:41, when $|\boldsymbol{v}|$ exceeds $700 \mathrm{~km} / \mathrm{s}$ ), the velocity moments reflect well collimated bulk flows. The flow reversal suggests that this is a classic example of an $\mathrm{x}$ line that moves tailward past the spacecraft.

Eastwood et al. (2005) pointed out that such a flow reversal may also be observed at an earthward moving o-line between two x-lines. They reported an event where they established the direction of motion of the flow reversal line by timeordering of the associated $B_{z}$ reversals at different satellites. 

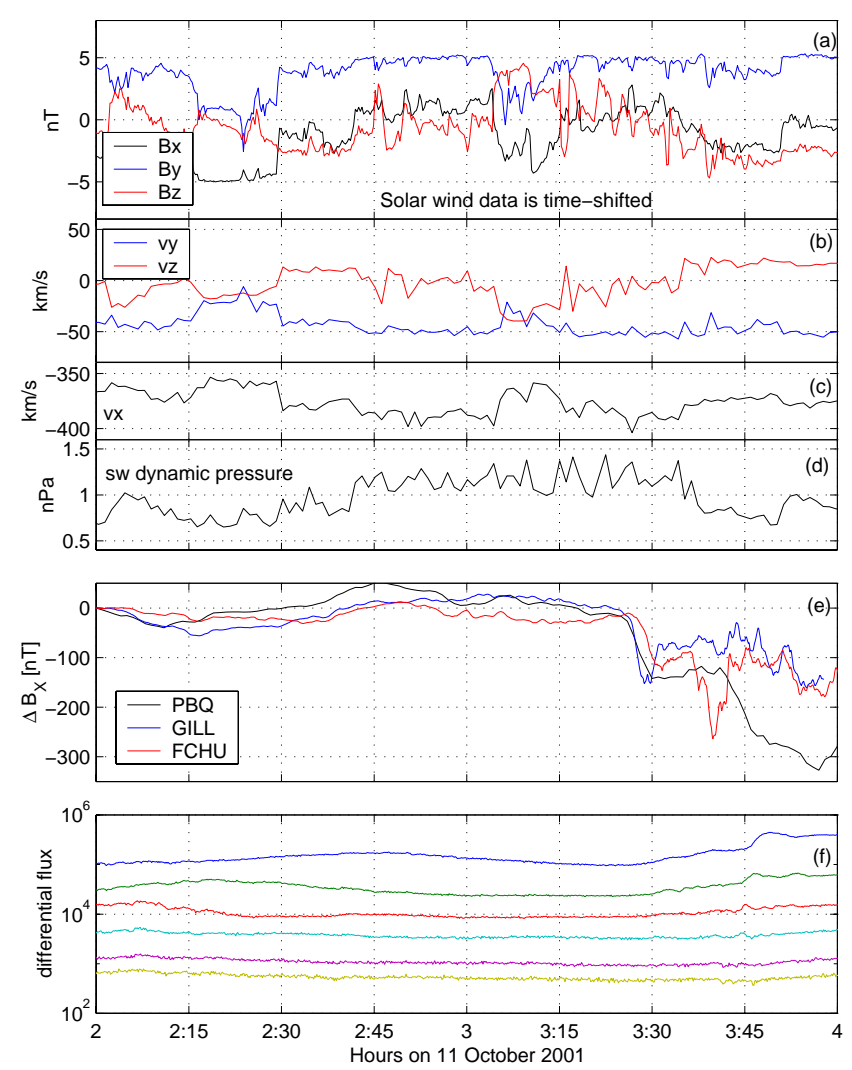

Fig. 2. (a-d) Solar wind conditions at 02:00-04:00 UT on 11 October 2001: components of the interplanetary magnetic field and solar wind velocity in GSM coordinates, and solar wind dynamic pressure. (e) Change in the northward (X) component of the magnetic field at three Canadian magnetometer stations: Poste de la Baleine (PBQ), Gillam (GILL) and Fort Churchill (FCHU). (f) LANL geosynchronous electron data: differential flux $\left(\mathrm{cm}^{-2} \mathrm{~s}^{-1} \mathrm{sr}^{-1} \mathrm{keV}^{-1}\right)$ of electrons recorded by the geosynchronous satellite 1990-095, which passed midnight meridian at 02:20 UT. The energy ranges of the curves are, from top to bottom: 50-75 keV, 75-105 keV, 105-150 keV, 150-225 keV, 225-315 keV and $315-500 \mathrm{keV}$.

Similar time-ordering unfortunately cannot be used in the present event. There is no distinguished major $B_{z}$ reversal, and the time-ordering of flow reversal at different satellites cannot be established, most likely because the spatial scale of the flow reversal region is much larger than the separation of the spacecraft. However, considering the lack of observation of other outflow jets than the two even though Cluster stays in the current sheet, and the clear Hall signature at the observed flow reversal (discussed in Sect. 3), the scenario of a tailward moving $\mathrm{x}$-line is best consistent with the observations.

From the $B_{x}$ component it is seen that Cluster reached the centre of the current sheet already before the start of the reconnection event, experienced several crossings during the
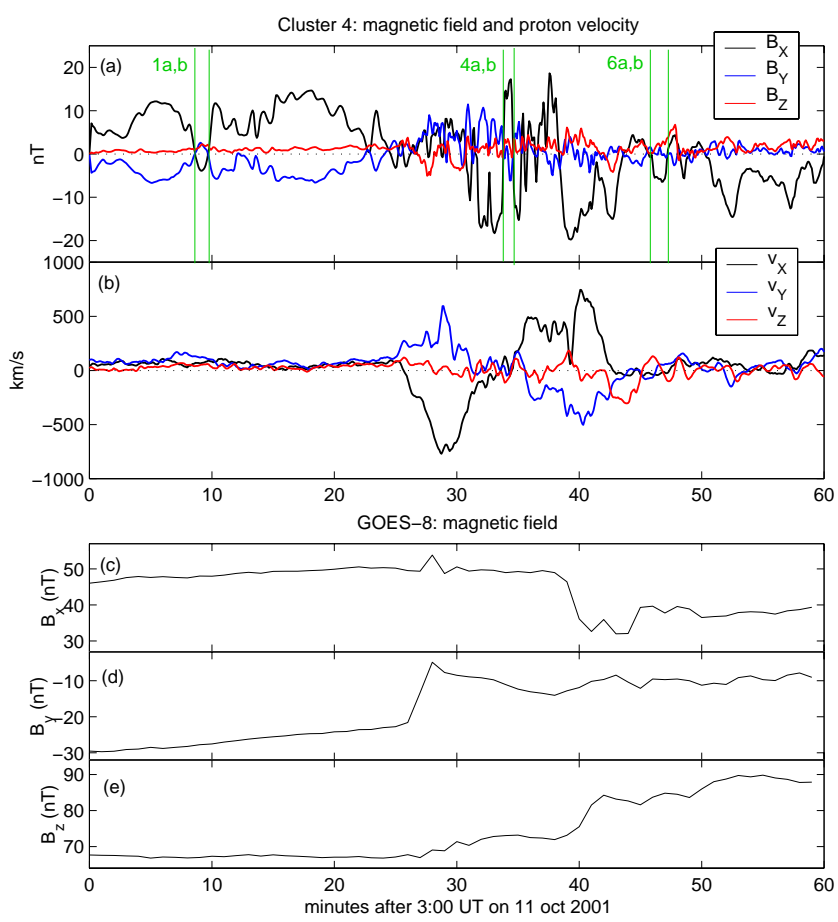

Fig. 3. Upper part: the components of magnetic field and proton velocity measured by Cluster 4, 03:00-04:00 UT on 11 October 2001, in GSM coordinates; magnetic field from FGM and proton velocity from CIS-CODIF. Lower part: the magnetic field components measured by Goes- 8 during the same interval.

event and remained within the current sheet even after it. The start of the tailward flow and the end of the earthward flow can be therefore interpreted as the start and end of reconnection - in this sector of the tail - and not just the satellites moving into or away from the current sheet. Thus, the observation suggests that the duration of the reconnection event was about $18 \mathrm{~min}$. In addition, there are some current sheet crossings both before and after the reconnection event. $B_{z}$ is weak but positive before the event, which probably means that the reconnection started on closed field lines.

The current sheet profiles during some of the crossings of this event were reconstructed by Runov et al. (2005). The profiles were single-peaked and in one case off-centered; current sheet bifurcation was not detected.

The lower part of Fig. 3 shows the magnetic field at geostationary orbit in the pre-midnight sector, recorded by Goes8. First there was a sudden change of about $15 \mathrm{nT}$ in $B_{y}$ at 03:26-03:28. Then about $12 \mathrm{~min}$ later, at 03:40, Goes- 8 recorded dipolarization. The time corresponds to an acceleration of earthward flow at Cluster, a short disturbance at Fort Churchill and the commencement of a longer deflection at Poste de la Baleine. The flux of energetic electrons (Fig. 2f) starts to increase soon after the onset of reconnection and shows a more rapid rise after the dipolarization at Goes-8. 
(a)

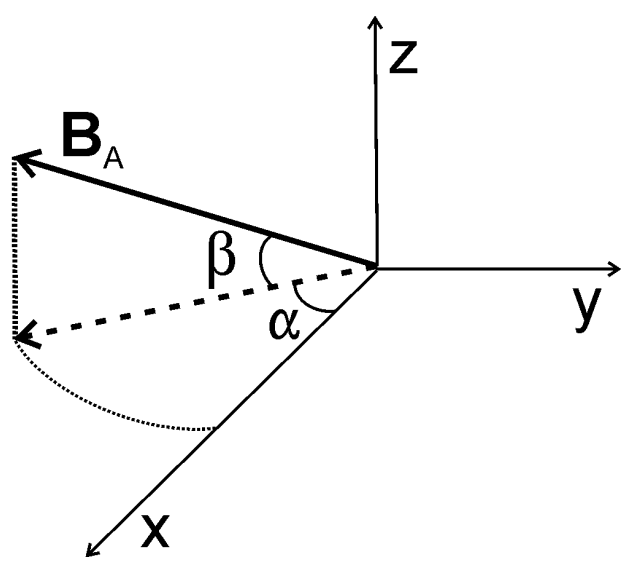

(b)

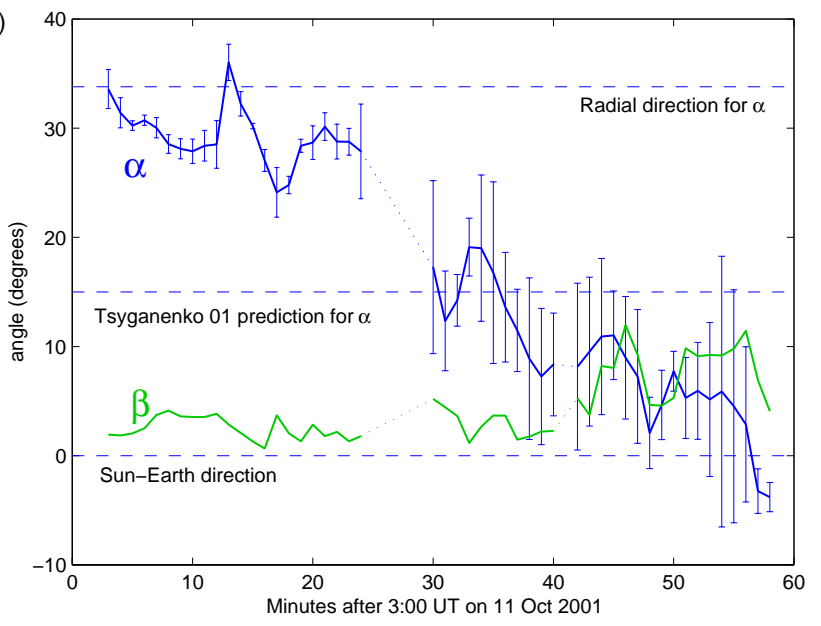

Fig. 4. The orientation of the ambient magnetic field at the Cluster site. (a) Definition of the angles $\alpha$ and $\beta$. The axes represent the GSM coordinate system. (b) The orientation of the ambient magnetic field as a function of time, obtained from minimum/maximum variance analysis. The lines are the averages of the results from the four spacecraft and the error bars show their standard deviation. Those data points where the standard deviation exceeds $20^{\circ}$ are omitted.

This suggests that there was an intensification of reconnection about $12 \mathrm{~min}$ after its first onset.

\subsection{Ambient field direction}

Figure 3 shows a very clear anticorrelation between $B_{x}$ and $B_{y}$ until about $03: 25$. Similarly the $\mathrm{x}$ and $\mathrm{y}$ components of proton velocity exhibit a clear anticorrelation during the outflows. After the event the correlation is not so clear.

Figure 4 examines more closely how the orientation of the ambient magnetic field changes. The two angles $\alpha$ and $\beta$ that describe the orientation are illustrated in the upper panel: $\beta$ is the angle between the ambient field and the $x y$ plane, and $\alpha$ is the angle between the $\mathrm{x}$-axis and the projection of ambient field in the $x y$ plane. In the lower panel the angles are calculated as a function of time using maximum/minimum variance analysis (MVA). For each data point, a five minutes long data interval was used to calculate the MVA eigenvectors. The angles were calculated from the eigenvector having the smallest angle with the $\mathrm{x}$-axis; in most cases this was the maximum variance eigenvector. The result is consistent with the average direction of the field itself during intervals when Cluster stays away from the central current sheet. However, long enough such intervals only occur before the start of reconnection and therefore MVA is needed to follow the time evolution of the ambient field direction.

The curves in Fig. $4 \mathrm{~b}$ show the average of the results from the four spacecraft, and the error bars in the $\alpha$ curve represent their standard deviation. The bars are omitted from the $\beta$ curve for clarity, but they would be similar in magnitude as those for $\alpha$. The deviations reflect short-timescale variations in the magnetic field direction near the current sheet centre, due to tilted orientations of the current sheet during oscillations. However, the purpose of Fig. $4 \mathrm{~b}$ is not to bring out all these variations but rather to assess the slow change in the background field on as global a scale as possible. Five minutes long data intervals were used in order to average out the effects of individual flappings.

Until 03:24 the four spacecraft give well consistent results: The $\alpha$ angle is rather large, about 30 degrees, and stays roughly constant with some minor variations. $\beta$ is very small, which means that the ambient field is quite accurately in the $x y$ plane. Between 03:24-03:29 the results from the four spacecraft are wildly varying and inconsistent with each other. This is because Cluster stayed in the central current sheet and did not see the ambient lobe field. After 03:29 the analysis produces again meaningful results, but the differences between the different spacecraft are now considerably larger reflecting the sub-Cluster-scale variations in the magnetic field. Despite the resulting inaccuracy one can see that $\alpha$ is now smaller and still decreases during the event.

One more caveat must be mentioned regarding the interpretation of $\alpha$ between 03:31-03:36. During this time the Hall fields may affect the analysis: earthward of the $\mathrm{x}$-line they tend to make $\alpha$ smaller, and they persisted in that sense for most of the period. However, overall one can conclude that before the onset of reconnection the ambient magnetic field was oriented approximately radially, but became almost aligned with the $\mathrm{x}$-axis during the event.

The geosynchronous satellite Goes- 8 was located roughly between Cluster and Earth in the same local time sector during the event (Fig. 1a). As shown in Fig. 3, Goes-8 recorded a sudden jump in the $B_{y}$ component, contemporaneous to the initial decrease in the $\alpha$ angle in Fig. 4. The orientation of the magnetic field in the $x y$ plane at the Goes- 8 location changed about 15 degrees in the same sense as at the Cluster location. This suggests that a large-scale configuration change took place in the magnetotail. Since the tail field orientation change coincides with the beginning of tail reconnection, as timed from the start of the tailward flow, it is probable that 
they are causally connected. This question will be discussed further in Sect. 5.1.

\subsection{Choosing the natural coordinate system}

The preceding analysis indicates that the GSM coordinate system is not very good for studying the event: a more natural coordinate system $x^{\prime} y^{\prime} z^{\prime}$ would be one in which the outflows and ambient lobe fields are aligned with the $x^{\prime}$ axis. As the ambient magnetic field direction changes during the event, the choice is ambiguous. To get another hint, we examine the proton bulk velocity. We find the smallest coordinate rotation angle $\alpha$ that removes the $v_{x}-v_{y}$ correlation in the time period 03:25-03:43, which is the shortest time interval including both the observed outflows. The results are given in the right column of Table 1 . The result stays the same even if the period 03:31-03:35, when the velocity moments are not relevant, is removed from the data. The table also gives angles of no correlation based on magnetic fields measurements. These have been calculated using the prereconnection interval 03:00-03:25.

It is interesting that although the orientation of the ambient lobe fields changes considerably during the reconnection event, the direction of both outflows is quite accurately that of the original ambient fields. This suggests that the local reconnection geometry may stay in the original orientation while global field orientation is already changing towards the $\mathrm{x}$-direction. We thus choose to align our basic coordinate system with the pre-reconnection ambient field, and rotate the GSM system 29 degrees clockwise around the $\mathrm{z}$-axis to accomplish that. Because the ambient field is very close to the $x y$ plane, we leave the $z^{\prime}$ axis to be the same as the $z$-axis.

\section{X-line}

Figure 5 gives an expanded view of the early and middle phases of the reconnection event. The bottom panel shows the components of the magnetic curvature vector. Its $\mathrm{x}$ component is negative, that is, the curvature is tailward until about 03:31:20, when strong earthward curvature is suddenly observed. After this initial spike the calculated curvature stays around zero for several minutes; this is because the entire Cluster constellation stays mostly on one or the other side of the current sheet centre, as evidenced by the $B_{x}$ components in panel (a). Each time when Cluster crosses the current sheet a "spike" of earthward curvature is observed. Our interpretation is that these spikes reflect a constant earthward curvature at the centre of the thin current sheet. Qualitatively the curvature data indicate that an $\mathrm{x}$-line passed Cluster at 03:31:20.

Corroborative evidence is provided by the Hall fields. Figure 6 illustrates the point by comparing statistically $B_{y^{\prime}}$ versus $B_{x^{\prime}}$. Until 03:30 UT no Hall signature is visible. Between 03:30 and 03:31 the data show a clear quadrupolar variation
Table 1. The angle of coordinate rotation in the $x y_{\mathrm{GSM}}$ plane that removes the correlation between $B_{x^{\prime}}$ and $B_{y^{\prime}}$ before the event, interval 03:00-03:25 UT, or between $v_{x^{\prime}}$ and $v_{y^{\prime}}$ during the event, interval 03:25-03:43 UT.

\begin{tabular}{lll}
\hline $\mathrm{s} / \mathrm{c}$ & $\boldsymbol{B}$ before & $\boldsymbol{v}$ during \\
\hline $\mathrm{C} 1$ & $28^{\circ}$ & $27^{\circ}$ \\
$\mathrm{C} 2$ & $30^{\circ}$ & $\mathrm{n} / \mathrm{a}$ \\
$\mathrm{C} 3$ & $29^{\circ}$ & $34^{\circ}$ \\
$\mathrm{C} 4$ & $28^{\circ}$ & $30^{\circ}$ \\
\hline
\end{tabular}

in $B_{y^{\prime}}$ (panel a). The sense of the variation is such that near the current sheet centre $B_{y^{\prime}}$ and $B_{x^{\prime}}$ anticorrelate, which corresponds to the tailward side of $\mathrm{x}$-line of the Hall structure. Then at 03:31:00 the Hall signature disappears for about 20 s (panel b). At 03:31:20 a clear Hall signature appears again (panel c), but its sense has been reversed and corresponds now to the earthward side of reconnection line.

During the next three minutes (panel d in Fig. 6) the statistical picture is somewhat cluttered because the current sheet undergoes flapping motions with strongly tilted orientations. It will be shown in the next subsection that the Hall structure is tilted with the current sheet during many of the crossings. At those instants the Hall signature is found mainly in $B_{z^{\prime}}$, and is thus not revealed by Fig. 6 . Also, some of the correlation visible in panel (d) may be due to the orientation change of the lobe fields as discussed in the previous section. However, to the extent that any Hall signature is observable, it retains its earthward of $\mathrm{x}$-line -sense during this period.

At 03:35-03:36 a clear and strong quadrupolar Hall signature is again observed (Fig. 6e). This coincides with the first rapid earthward acceleration of protons (Fig. 5d). After 03:36 a strong correlation between $B_{x^{\prime}}$ and $B_{y^{\prime}}$ remains and persists also after the time interval shown, but it is due to the ambient field orientation change discussed in Sect. 2.2. We thus interpret the interval 03:30-03:36 as the time that Cluster spent in the diffusion region, because during this interval it was between the outflow jets and recorded Hall signatures. Only one x-line passing was observed at about 3:31, but the possibility of multiple $\mathrm{x}$-lines cannot be completely ruled out.

\section{Current sheet crossings}

\subsection{Before, during and after reconnection}

Repeated rapid current sheet crossings have been observed frequently in the tail. A study by Sergeev et al. (2004) showed that they are due to oscillations that propagate mainly towards the flanks from the centre of the tail. The interval 03:00-04:00 UT also shows such oscillations, which are particularly intense during the reconnection event. 


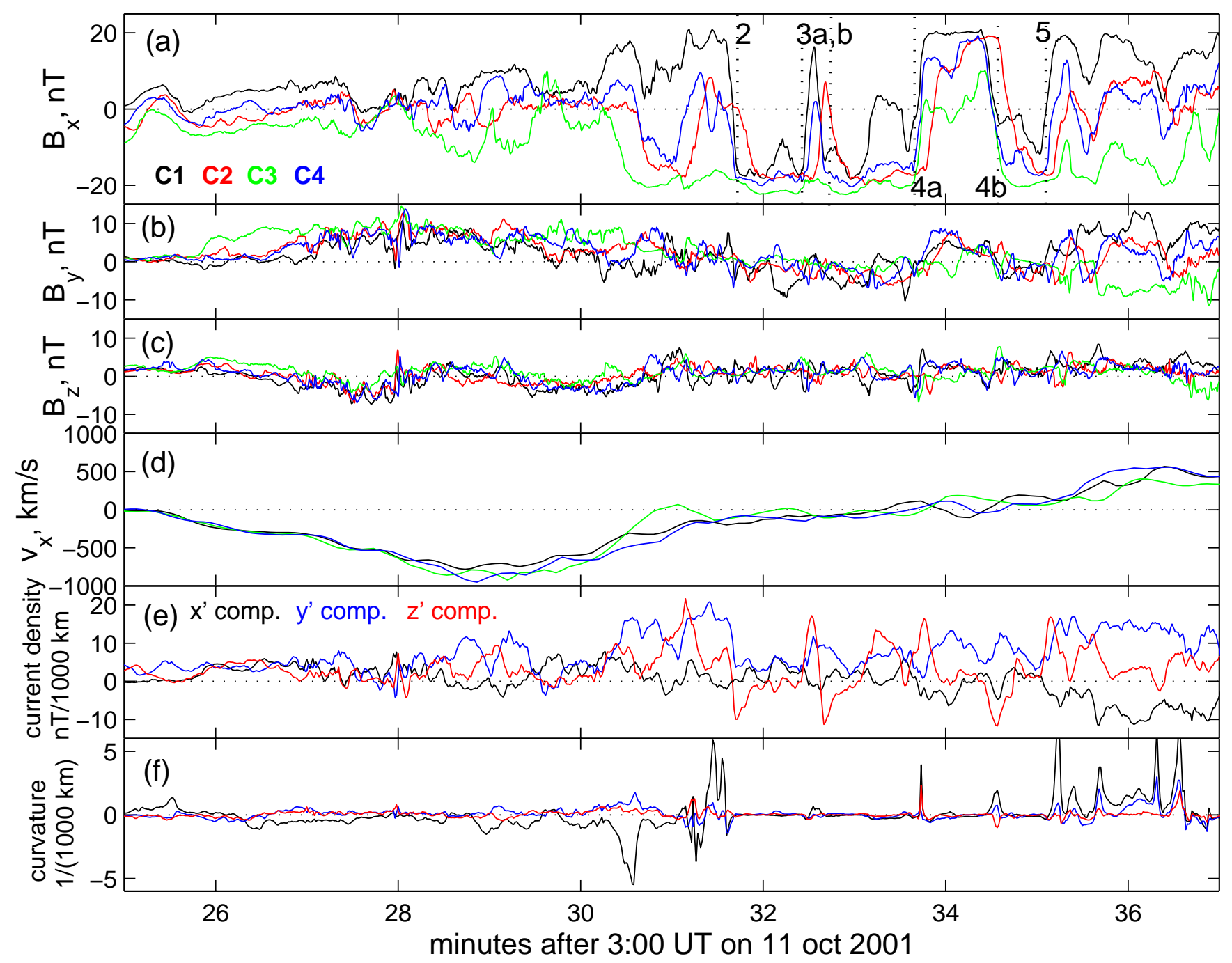

Fig. 5. Observed and calculated quantities during the event, given in the $x^{\prime} y^{\prime} z^{\prime}$ coordinate system defined in Sect. 2.4. (a-c) Magnetic field components. Data from Cluster 1 in black, C2 in red, C3 in green and C4 in blue. (d) x component of proton flow velocity, colours as in $(\mathrm{a}-\mathrm{c})$. Proton data from $\mathrm{C} 2$ is missing because its CIS instrument group is broken. (e) Current density and (f) curvature vector at the Cluster barycentre. $x^{\prime}$ component in black, $y^{\prime}$ in blue and $z^{\prime}$ in red.

On three occasions an oscillation passes all the four Cluster spacecraft so that the orientation and normal velocity of the moving current sheet can be determined by multi-point timing analysis. The crossing pairs are marked in Fig. 3, pair 4 also in Fig. 5. Table 2 shows the timing results. The validity of the current sheet normal directions was checked by MVA and by comparing them to the current density vectors given by the curlometer method (Dunlop et al., 2002).

The normal velocities are remarkably similar within each pair of crossings. Around 03:09 when the current sheet is in a relatively quiet state, it passes Cluster first upward and later downward with a velocity of about $60 \mathrm{~km} / \mathrm{s}$. The next double crossing (number 4) occurs during reconnection, in the thin current sheet of the diffusion region, and has speeds of about $170 \mathrm{~km} / \mathrm{s}$. The crossings 6 then take place when the reconnection has ceased but the current sheet is still in a disturbed state. Now the current sheet motion is a bit slower than during reconnection, about $120 \mathrm{~km} / \mathrm{s}$. These findings are in good accordance with the statistical result of Sergeev et al. (2004), who state that the average propagation speed is $57 \mathrm{~km} / \mathrm{s}$ in quiet current sheets and $145 \mathrm{~km} / \mathrm{s}$ in active sheets.

Although only three double crossings are suitable for timing analysis, they do not represent isolated waves. The first double crossing is followed by a series of smaller oscillations with a period of about $1.5 \mathrm{~min}$, as can be seen from the $B_{x}$ data in Fig. 3. In the thinner current sheet during reconnection the oscillations are quite irregular, but seem to have a period of about $1 \mathrm{~min}$ (Fig. 5). Cluster 2 records all 

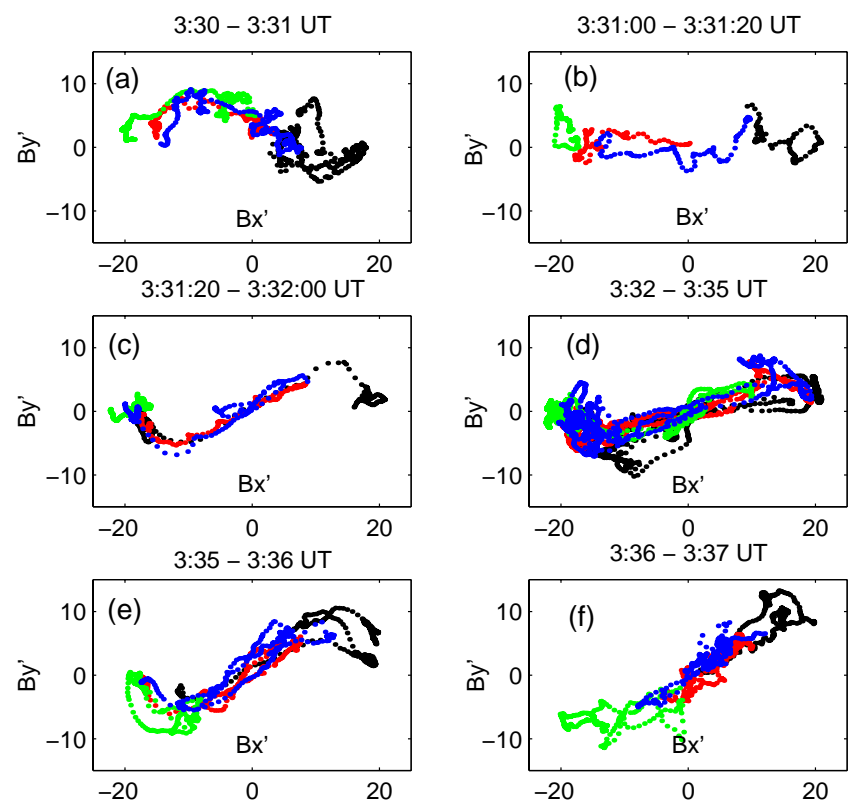

Fig. 6. $B_{y^{\prime}}$ versus $B_{x^{\prime}}$ in six consecutive time intervals during the reconnection event, illustrating the Hall effect. The colours refer to different spacecraft: data from Cluster 1 is in black, C2 in red, C3 in green and $\mathrm{C} 4$ in blue.

Table 2. The normal vector and the normal velocity of the current sheet in three pairs of crossings, marked in Fig. 3. The normal vector components are given in GSM $([x, y, z])$ and rotated $\left(\left[x^{\prime}, y^{\prime}, z^{\prime}\right]\right)$ coordinates. The results have been obtained by timing analysis.

\begin{tabular}{rrrr}
\hline & {$\left[n_{x}, n_{y}, n_{z}\right]$} & {$\left[n_{x^{\prime}}, n_{y^{\prime}}, n_{z^{\prime}}\right]$} & $v_{n}(\mathrm{~km} / \mathrm{s})$ \\
\hline 1a & {$[.22,-.02, .97]$} & {$[.20,-.09, .97]$} & 56 \\
1b & {$[.09, .86,-.50]$} & {$[-.33, .80,-.50]$} & 60 \\
\hline 4a & {$[.32, .94, .10]$} & {$[-.18, .98, .10]$} & 177 \\
4b & {$[.49, .67, .56]$} & {$[.10, .82, .56]$} & 157 \\
\hline 6a & {$[.05, .32, .95]$} & {$[-.11, .30, .95]$} & 118 \\
6b & {$[-.24, .97, .01]$} & {$[-.68, .73, .01]$} & 117 \\
\hline
\end{tabular}

these oscillations about $10 \mathrm{~s}$ after the other three spacecraft, and as Cluster 2 is located about $1000 \mathrm{~km}$ duskward of the other satellites (Fig. 1b), this indicates that the oscillations are travelling duskward with an order-of-magnitude velocity of $100 \mathrm{~km} / \mathrm{s}$. Together with the observed period this leads to the order-of-magnitude estimate of $1 R_{E}$ for the wavelength of the oscillations.

\section{$4.2 B_{z^{\prime}}$ variations from tilted Hall fields}

The $y^{\prime}$ and $z^{\prime}$ components of the magnetic field show rather strong small-scale fluctuations during the entire event. Many
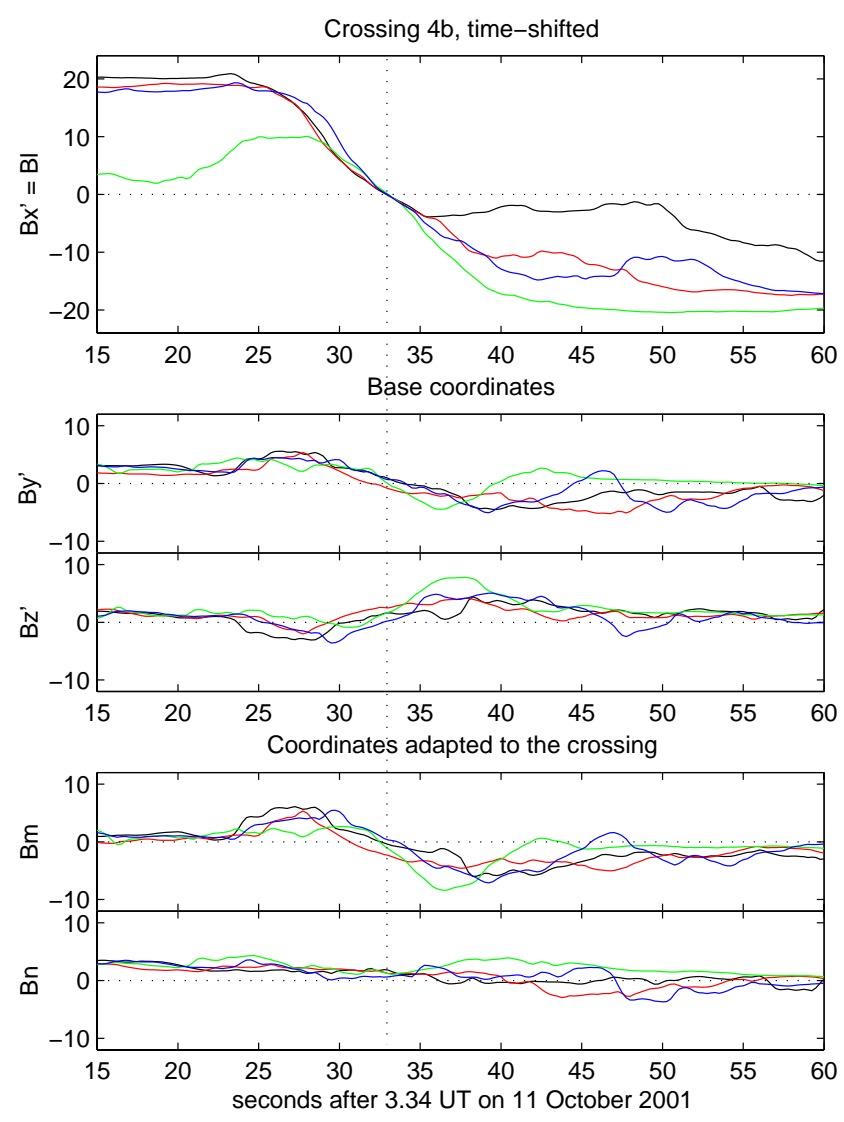

Fig. 7. The magnetic field components measured by Cluster during the crossing $4 \mathrm{~b}$ in Fig. 5. The data have been time-shifted so that the $B_{x^{\prime}}=B_{l}$ sign changes coincide; the time axis gives real time for $\mathrm{C}$. In the two lowermost panels the coordinates have been rotated to show the Hall effect in $B_{m}$. Colouring of the lines as in Figs. 5a-c and 6.

of the strongest fluctuations in $B_{z^{\prime}}$ coincide with $B_{x^{\prime}}$ sign changes and consist of a negative peak followed immediately by a positive one. Figure 7 examines in detail one such crossing, $4 \mathrm{~b}$ in Table 2 and Fig. 5. The data from Cluster 2, 3 and 4 are time-shifted so that the $B_{x^{\prime}}$ sign changes coincide.

The first three panels show the magnetic field components during the crossing in the coordinates $x^{\prime} y^{\prime} z^{\prime}$, same as in Fig. 5. Bipolar variation is seen in both $B_{y^{\prime}}$ and $B_{z^{\prime}}$. Then another rotation of $43^{\circ}$, in a left-handed sense around the $x^{\prime}$ axis, was applied to the coordinate axes. The angle was chosen to remove correlation between the second and third magnetic field components, denoted $B_{m}$ and $B_{n}$ in these new coordinates $l m n$. Table 3 gives the orientations of these axes in GSM coordinates. The $n$ axis is approximately aligned with the current sheet normal given by timing analysis (cf. Table 2); the cosine of their angle is $0.97 . B_{m}$ and $B_{n}$ are shown in the two last panels of Fig. 5. 
Table 3. The unit base vectors of the two specialised coordinate systems $x^{\prime} y^{\prime} z^{\prime}$ and $l m n$, given in GSM coordinates.

\begin{tabular}{lrrr}
\hline & $x_{\mathrm{GSM}}$ & $y_{\mathrm{GSM}}$ & $z_{\mathrm{GSM}}$ \\
\hline$\hat{\boldsymbol{x}}^{\prime}$ & 0.875 & -0.485 & 0 \\
$\hat{\boldsymbol{y}}^{\prime}$ & 0.485 & 0.875 & 0 \\
$\hat{\boldsymbol{z}}^{\prime}$ & 0 & 0 & 1 \\
\hline$\hat{\boldsymbol{l}}$ & 0.875 & -0.485 & 0 \\
$\hat{\boldsymbol{m}}$ & 0.355 & 0.640 & -0.682 \\
$\hat{\boldsymbol{n}}$ & 0.331 & 0.596 & 0.731 \\
\hline
\end{tabular}

In the new coordinate system there is almost no variation in $B_{n}$ until 03:34:35, which confirms that the chosen coordinate system is well fitted to the crossing. The Hall signature was recognizable already in $B_{y^{\prime}}$, but in $B_{m}$ it is stronger and clearer. Note especially the data of Cluster 3 (green) after crossing the current sheet centre: $\left|B_{m}\right|$ increases directly proportionally to $\left|B_{l}\right|$ until $B_{l}$ reaches about $-10 \mathrm{nT}$. When Cluster 3 travels farther into the southern lobe, $\left|B_{m}\right|$ decreases and vanishes when $B_{l}$ reaches about $-20 \mathrm{nT}$. The other three satellites stay closer to the current sheet centre, and consequently their measured $B_{m}$ values stay negative considerably longer after the crossing.

We searched for Hall effects also from all the other crossings 2-5 marked in Fig. 5. We used both correlationminimizing rotations around the $x^{\prime}$ axis only and MVA coordinates. Clear Hall effects were found from all the crossings except $3 a$ and $3 b$, in which the Hall fields were only barely recognizable. The double crossing 3 was more difficult to treat than the others, because three of the spacecraft crossed the current sheet only half-way and the return to the southern side was immediate (Cluster 3 did not cross at all).

In a majority of the crossings the coordinate transformation that brought out the Hall fields in the second $(m)$ component of $\boldsymbol{B}$ also made variation in the third (n) component of $\boldsymbol{B}$ relatively small. This was the case in crossings 2, 3a, $4 \mathrm{~b}$ and 5. In $3 \mathrm{~b}$ and $4 \mathrm{a}$ there remain variations in $B_{n}$ that are about as large as the Hall effect in $B_{m}$. We thus conclude the following: during the reconnection event under examination Cluster stays several minutes in the diffusion region and sees there strong wave-like oscillations of the current sheet, which propagate duskward. Hall fields can be recognized in connection with the crossings, and they are in an orientation tilted along the current sheet. This explains most of the largest fluctuations in $B_{z^{\prime}}$, but in addition to flapping and Hall fields there is considerable irregular small-scale variation in the magnetic field.

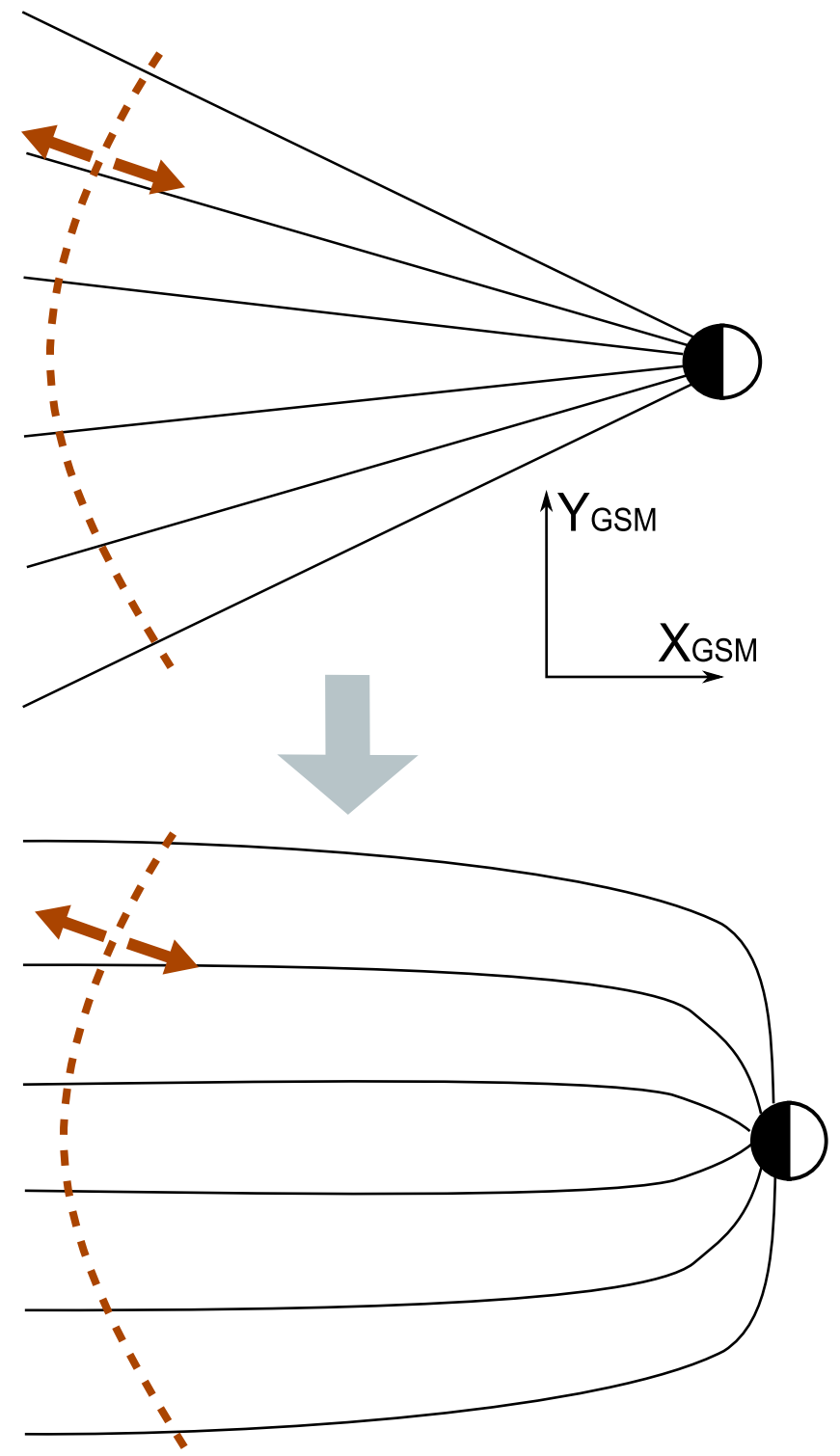

Fig. 8. A schematic illustration of how the orientation of the magnetic field in the tail may have changed due to the reconnection event. An x-line and outflows are also drawn. The figure shows a view looking down on the $x y$ plane.

\section{Discussion and conclusions}

5.1 A global configuration change in the tail due to reconnection?

As was shown in Sect. 2, the orientation of the ambient lobe fields near the current sheet in the magnetotail changed about $15^{\circ}$ soon after the onset of reconnection. The change was recorded by both Cluster and Goes- 8 in the premidnight magnetotail, and at the Cluster site the change continued gradually during the entire reconnection period. The 
deflection thus signifies a large scale configuration change in the tail. At the time there were no other satellites that could provide magnetometer data from the magnetotail, so we do not know whether this configuration change was truly global and affected also the post-midnight part of the tail. Figure 8 that illustrates the configuration change has been drawn assuming symmetry in the y-direction.

Solar wind data from the ACE and WIND satellites show that this change was not caused directly by the solar wind; in particular, the IMF y-component stays almost constant at $+4 \ldots+5 \mathrm{nT}$ during the entire event. The solar wind velocity and the dynamic pressure also show only slight variations (see Fig. 2). IMF z-component changed from positive to negative around 03:20 UT, but the change was gradual and too slow to be considered as the direct cause of the observed fast orientation change. The change was therefore most probably associated with the reconnection that started at the same time.

Simultaneous ground signatures were also observed by three Canadian magnetometer stations. A southward deflection of about $150 \mathrm{nT}$ was recorded at Gillam and Poste de la Baleine at 03:27 UT - at the same time when Goes- 8 observed the deflection in $B_{y}$ - and a couple of minutes later also at Fort Churchill (Fig. 2e). The magnetic signature was a clear indication of a westward electrojet. Gillam and Poste de la Baleine have the same magnetic latitude but are separated by $25^{\circ}$ in longitude, Gillam being the more western station, while Fort Churchill is $2.3^{\circ}$ to the north from Gillam. The disturbance was thus propagating northward but did not advance to the stations north of Fort Churchill. Nor was it observed on more eastern or western stations. According to the Tsyganenko 96 model Fort Churchill was near to the magnetic footpoint of Cluster and Poste de la Baleine near to that of Goes-8. The question is thus left open, whether this phenomenon was tail-wide or limited to the sector in which Cluster and Goes-8 were.

The Tsyganenko 2001 model predicts that the angle between the magnetic field and $\mathrm{x}$-axis at the Goes- 8 location should be $22^{\circ}$. This is consistent with the measured angle before the deflection at 03:27. At the Cluster location T01 predicts an ambient field angle of about $15^{\circ}$, which is consistent with the MVA result at 03:30, immediately after the initial deflection but before the subsequent gradual change. Thus, before the onset of reconnection the tail widens at the Cluster site more than the model predicts, but during the reconnection event the field both at the Cluster and Goes- 8 sites becomes more aligned with the $\mathrm{x}$-axis than in the model.

What may have caused this tail configuration change? It was not compression by solar wind or penetration of IMF y-component. Dipolarization also occurs at Goes-8, but only about 12 min later, so this was something different. The onset of reconnection (timed from the start of the tailward flow at Cluster), the configuration change at Cluster and at Goes8 , and the commencement of a westward electrojet in the ionosphere were all practically simultaneous: time-ordering cannot be established from the observations.

We hypothesize that a large-scale instability involving field-aligned currents coupling to the ionosphere is responsible for the onset of reconnection and the simultaneous configuration change. Either release of stress associated with the current disruption or the tailward motion of the $\mathrm{x}$-line then led to the field rotation from radial to one aligned with the Sun-Earth line. Reconnection also caused flux to pile up in the earthward side causing the field dipolarization.

\section{2 $\mathrm{X}$-line at the edge of the diffusion region}

In Sect. 3 we showed that Cluster was in the diffusion region between the outflow jets during 03:30-03:36 and that an $\mathrm{x}$-line passed Cluster at about 03:31, that is, much sooner than halfway in the diffusion region. There are at least four possible reasons to this:

1. The $x$-line was near the edge of the diffusion region. In that case the x-line "leads" the tailward motion of the reconnection and "drags" the diffusion region behind. Protons become demagnetized for a relatively long time in those regions through which the $\mathrm{x}$-line has passed.

2. The diffusion region is symmetric but spreads out as the reconnection site grows older. Cluster recorded the tailward half of the diffusion region soon after the onset of reconnection, but when it was in the earthward half the diffusion region grew so much wider that it took much longer before its earthward edge reached Cluster.

3. The reconnection region moves tailward at a changing speed or stepwise. In this event, it moved first quickly but then just happened to stop for a few minutes when the $\mathrm{x}$-line had passed Cluster. The reconnection region may have stopped another time at 03:36-03:39, which would explain the apparent two steps in the earthward acceleration (see Fig. 3).

4. The diffusion region had multiple $x$-lines, but only the most tailward one happened to be clearly observable in the magnetometer recordings. The other $\mathrm{x}$-line(s) may have passed when Cluster was away from the current sheet centre.

Decisive discrimination between these hypotheses would require observing the reconnection site at two or more different $x$ values on its way tailward.

\subsection{Hall fields conform to current sheet kinks}

During the event the current sheet exhibits strong flapping motions that propagate toward the flank of the tail. The oscillations are rather irregular in amplitude but have a period of about $1.5 \mathrm{~min}$ before the onset of reconnection and $1 \mathrm{~min}$ during reconnection. The speed of the oscillations changes 


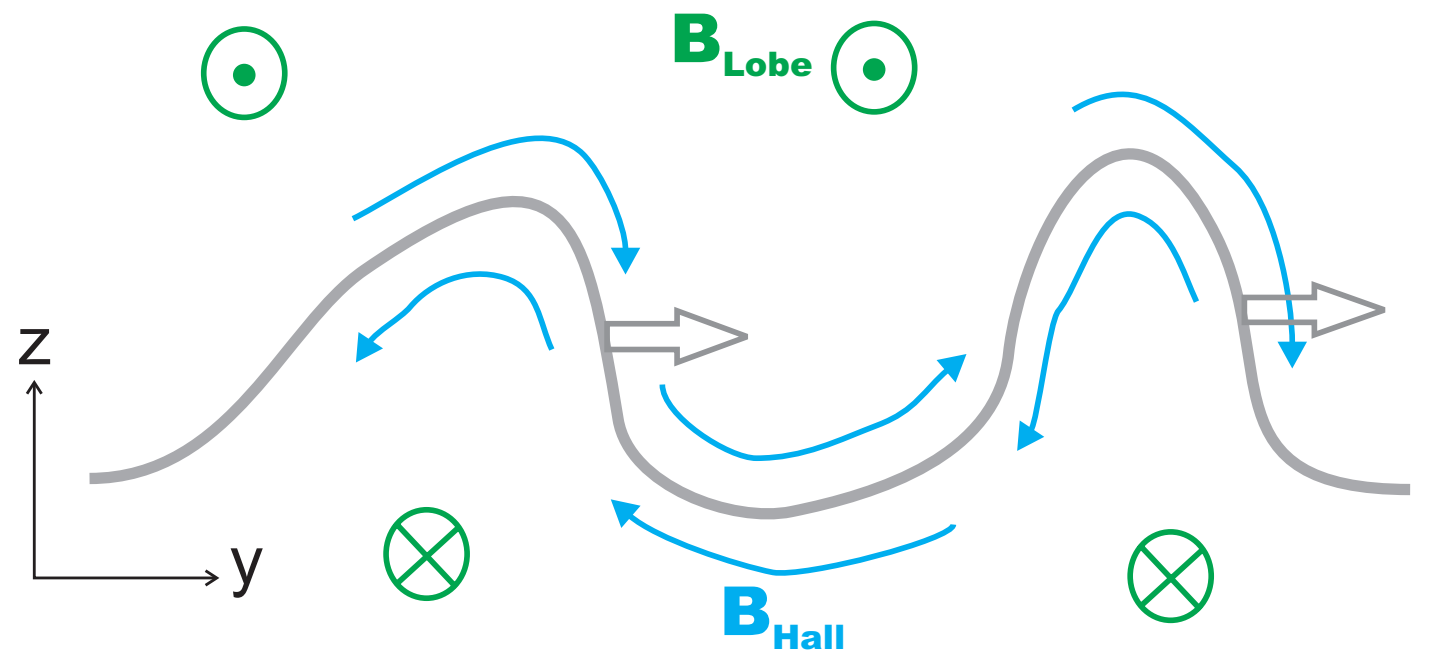

Fig. 9. A schematic illustration of duskward-propagating kinks in the current sheet. The ambient magnetic field direction is shown in green, while the Hall fields are in blue. The Hall fields stay adjacent to the current sheet and conform to its kinks.

when the current sheet turns from quiet to disturbed state: before reconnection onset the current sheet passes Cluster at a speed of about $60 \mathrm{~km} / \mathrm{s}$, during reconnection at $170 \mathrm{~km} / \mathrm{s}$ and after reconnection at $120 \mathrm{~km} / \mathrm{s}$. The current sheet normal has a large $y^{\prime}$ component during most of these crossings, indicating that the current sheet is strongly tilted away from the $x y$ plane.

There are many quite large-amplitude fluctuations in $B_{z^{\prime}}$ during this reconnection event, especially within the diffusion region (Fig. 5). That kind of fluctuations are often interpreted as signatures of plasmoids or flux ropes, or said to signify filamentation of the cross-tail current associated with current disruption model (see, e.g., Lui et al., 2005). However, it was shown in Sect. 4.2 that in this event most of the largest fluctuations are caused by Hall fields. They appear as variations in $B_{z^{\prime}}$, instead of only in $B_{y^{\prime}}$, because in many crossings the current sheet is in a strongly tilted orientation. This also indicates that examination of $B_{y}$ is not always sufficient to establish the presence or absence of Hall fields. Figure 9 illustrates how the quadrupolar Hall structure conforms to the waves propagating in the current sheet.

Acknowledgements. We thank H. Singer at NOAA SEC for the GOES-8 magnetometer key parameter data, N. Ness at Bartol Research Institute for ACE magnetometer data, and D. J. McComas at Southwest Research Institute for ACE SWEPAM data. These satellite data were obtained through the CDAWeb. We also acknowledge D. Belian (PI) and G. D. Reeves for providing the LANL SOPA energetic electron data, Canadian Space Agency for the GILL and FCHU magnetometer data and Geological Survey of Canada for the PBQ magnetometer data.

T. V. Laitinen thanks N. Partamies for help with the ground magnetometer data, T. I. Pulkkinen for reading and commenting the manuscript, N. Ganushkina for help with the Tsyganenko models, and K. Kauristie, M. Palmroth and H. Koskinen for inspiring dis- cussions in Finnish. The work of T. V. Laitinen was supported by the Magnus Ehrnrooth foundation and his visit to the IWF by the Vilho, Yrjö and Kalle Väisälä foundation.

Topical Editor I. A. Daglis thanks L. Zelenyi and A. Vaivads for their help in evaluating this paper.

\section{References}

Asano, Y., Mukai, T., Hoshino, M., Saito, Y., Hayakawa, H., and Nagai, T.: Evolution of the thin current sheet in a substorm observed by Geotail, J. Geophys. Res., 108(A5), 1189, doi:10.1029/2002JA009785, 2003.

Baker, D. N., Pulkkinen, T. I., Angelopoulos, V., Baumjohann, W., and McPherron, R. L.: The neutral line model of substorms: Past results and present view, J. Geophys. Res., 101, 12 975-13010, 1996.

Balogh, A., Carr, C. M., Acuña, M. H., et al.: The Cluster Magnetic Field Investigation: overview of in-flight performance and initial results, Ann. Geophys., 19, 1207-1217, 2001, http://www.ann-geophys.net/19/1207/2001/.

Baumjohann, W., Paschmann, G., and Luhr, H.: Characteristics of high-speed ion flows in the plasma sheet, J. Geophys. Res., 95, 3801-3809, 1990.

Belehaki, A., McEntire, R. W., Kokubun, S., and Yamamoto, T.: Magnetotail response during a strong substorm as observed by GEOTAIL in the distant tail, Ann. Geophys. 16, 528-541, 1998.

Deng, X. H., Matsumoto, H., Kojima, H., Mukai, T., Anderson, R. R., Baumjohann, W., and Nakamura, R.: Geotail encounter with reconnection diffusion region in the Earth's magnetotail: Evidence of multiple $\mathrm{X}$ lines collisionless reconnection?, J. Geophys. Res., 109, A05206, doi:10.1029/2003JA010031, 2004.

Dunlop, M. W., Balogh, A., Glassmeier, K.-H., and Robert, B.: Four-point Cluster application of magnetic field analysis tools: The Curlometer, J. Geophys. Res., 107(A11), 1384, doi:10.1029/2001JA005088, 2002. 
Eastwood, J. P., Sibeck, D. G., Slavin, J. A., Goldstein, M. L., Lavraud, B., Sitnov, M., Imber, S., Balogh, A., Lucek, E. A., and Dandouras, I.: Observations of multiple X-line structure in the Earth's magnetotail current sheet: A Cluster case study, Geophys. Res. Lett. 32, L11105, doi:10.1029/2005GL022509, 2005.

Hones Jr., E. W., Birn, J., Baker, D. N., Bame, S. J., Feldman, W. C., McComas, D. J., Zwickl, R. D., Slavin, J. A., Smith, E. J., and Tsurutani, B. T.: Detailed examination of a plasmoid in the distant magnetotail with ISEE 3, Geophys. Res. Lett. 11, 10461049, 1984.

Hoshino, M., Nishida, A., Mukai, T., Saito, Y., Yamamoto, T., and Kokubun, S.: Structure of plasma sheet in magnetotail: Doublepeaked electric current sheet, J. Geophys. Res., 101, 24775$24786,1996$.

Laitinen, T. V., Pulkkinen, T.I., Palmroth, M., Janhunen, P., and Koskinen, H. E. J.: The magnetotail reconnection region in a global MHD simulation, Ann. Geophys., 23, 3753-3764, 2005, http://www.ann-geophys.net/23/3753/2005/.

Lui, A. T. Y, Meng, C.-I., and Akasofu, S.-I.: Wavy nature of the magnetotail neutral sheet, Geophys. Res. Lett., 5, 279-282, 1978.

Lui, A. T. Y., Jacquey, C., Lakhina, G. S., Lundin, R., Nagai, T., Phan, T.-D., Pu, Z. Y., Roth, M., Song, Y., Treumann, R. A., Yamauchi, M., and Zelenyi, L. M.: Critical issues on magnetic reconnection in space plasmas, Space Sci. Rev., 116, 477-521, doi:10.1007/s11214-005-1987-6, 2005.

Nagai, T.: Location of magnetic reconnection in the magnetotail, Space Sci. Rev., 122, 39-54, doi:10.1007/s11214-006-6216-4, 2006.

Pulkkinen, T. I., Palmroth, M., Tanskanen, E. I., Janhunen, P., Koskinen, H. E. J., and Laitinen, T. V.: New interpretation of magnetospheric energy circulation, Geophys. Res. Lett., 33, L07101, doi:10.1029/2005GL025457, 2006.
Rème, H., Aoustin, C., Bosqued, J. M., et al.: First multispacecraft ion measurements in and near the Earth's magnetosphere with the identical Cluster ion spectrometry (CIS) experiment, Ann. Geophys., 19, 1303-1354, 2001, http://www.ann-geophys.net/19/1303/2001/.

Runov, A., Sergeev, V.A., Nakamura, R., Baumjohann, W., Zhang, T.L., Asano, Y., Volwerk, M., Vörös, Z., Balogh, A., and Rème, H.: Reconstruction of the magnetotail current sheet structure using multi-point Cluster measurements, Planet. Space Sci., 53, 237-243, 2005.

Sergeev, V. A., Semenov, V. S., and Sidneva, M. V.: Impulsive reconnection in the magnetotail during substorm expansion, Planet Space Sci. 35, 1199-1212, 1987.

Sergeev, V., Runov, A., Baumjohann, W., Nakamura, R., Zhang, T. L., Volwerk, M., Balogh, A., Rème, H., Sauvaud, J. A., André, M., and Klecker, B.: Current sheet flapping motion and structure observed by Cluster, Geophys. Res. Lett. 30, 1327-1330, doi:10.1029/2002GL016500, 2003.

Sergeev, V., Runov, A., Baumjohann, W., Nakamura, R., Zhang, T. L., Balogh, A., Louarnd, P., Sauvaud, J.-A., and Rème, H.: Orientation and propagation of current sheet oscillations, Geophys. Res. Lett. 31, L05807, doi:10.1029/2003GL019346, 2004.

Shay, M. A., Drake, J. F., Swisdak, M., Dorland, W., and Rogers, B. N.: Inherently three dimensional magnetic reconnection: A mechanism for bursty bulk flows?, Geophys. Res. Lett., 30(6), 1345, doi:10.1029/2002GL016267, 2003.

Sonnerup, B. U. Ö.: Magnetic reconnection, in Solar System Plasma Physics, vol. 3, edited by: Lanzerotti, L. J., Kennel, C. F., and Parker, E. N., pp. 45-108, North-Holland, New York, 1979.

Thompson, S. M., Kivelson, M. G., El-Alaoui, M., Balogh, A., Rème, H., and Kistler, L. M.: Bifurcated current sheets: Statistics from Cluster magnetometer measurements, J. Geophys. Res. 111, A03212, doi:10.1029/2005JA011009, 2006. 